\title{
Public reports of infection rates urged
}

Previously published at www.cmaj.ca

$\mathrm{M}$ any Canadian hospitals have not set the bar high enough in the battle against Clostridium difficile, says the head of the Canadian Patient Safety Institute.

"I think we sometimes set a very mediocre standard for ourselves. We look around and see what the average is and say that is the standard," says Phil Hassen, executive director of the institute.

Shining a very public spotlight on $C$. difficile cases in individual hospitals will probably be necessary to reduce rates of the potentially deadly infection, adds Hassen. Public reporting "causes everyone to pay attention."

It's an approach that has merit, says Pamela Fralick, president of the Canadian Healthcare Association. "We are talking about patient safety and you can't worry about hurting an institution's feelings. You can't spare the institution at the expense of the patients."

Fralick would, however, like to see outcome data from Ontario, where hospital rates of $C$. difficile are now posted publicly. Still, she adds, "look at the numbers and we really should be paying more attention."

$C$. difficile reporting is now mandatory in only four provinces - Ontario, Quebec, Manitoba and Newfoundland and Labrador - and individual hospital rates are made public only in Ontario and Quebec.

Long considered a routine, almost inevitable, part of institutional culture, the risks posed by hospital-acquired $C$. difficile infections were propelled into public consciousness after the 20022006 outbreak in Quebec that claimed about 1500 lives.

One in 10 adults contract an infection while in hospital and $C$. difficile mortality rates remain sobering. Thirty days after contracting $C$. difficile, mortality rates for patients are 17.9 per 100 in Ontario and Quebec, 15.1 in Atlantic Canada and 10.7 in the western provinces, according to the latest (2007)

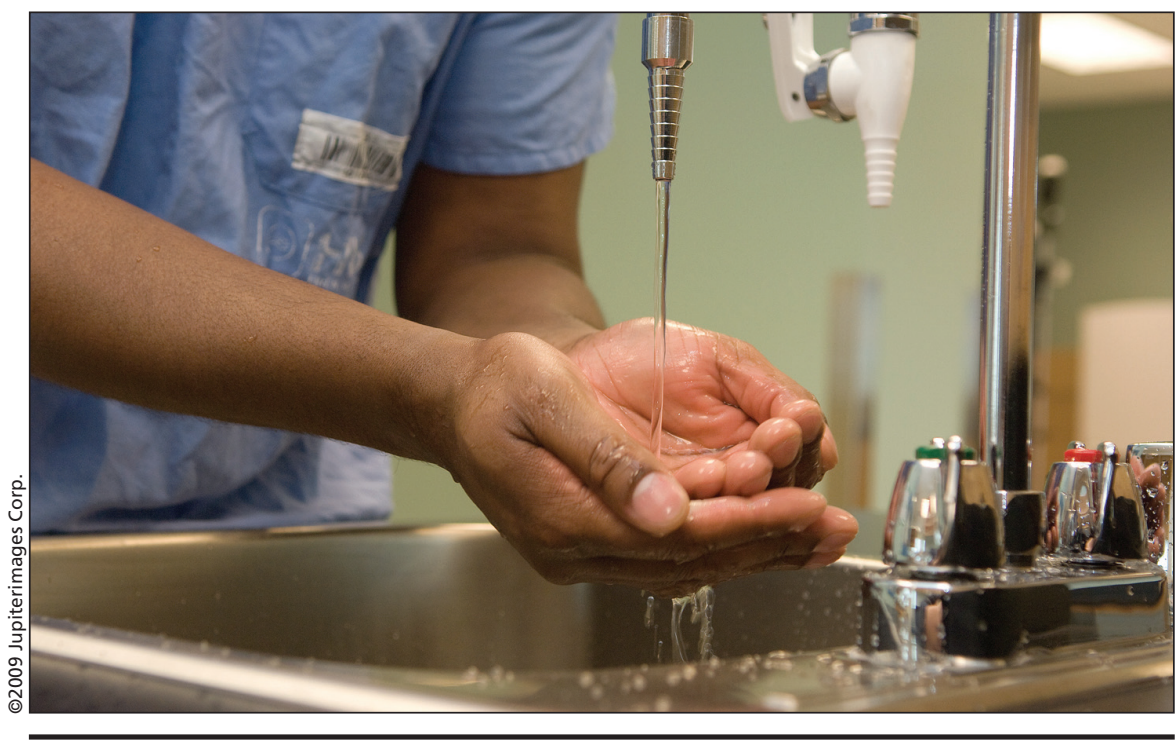

Handwashing will have little impact on hospital C. difficile rates if it isn't accompanied by other institutional measures such as improved cleaning regimes, experts say.

figures from the Canadian Nosocomial Infection Surveillance Program.

Yet, however deadly $C$. difficile infections can be, they are still considered "far less of a threat to patient safety than adverse events such as medication administration errors and falls," Dr. Michael Gardam, director of infectious disease prevention and control at the Ontario Agency for Health Protection and Promotion, and several colleagues recently asserted (HealthcarePapers 2009;9:8-24).

Hassen wants that to change, and says reducing rates of hospital-acquired infections is one of the top three initiatives needed to improve patient safety in Canada.

A concentrated effort can achieve success, he adds. For example, it used to be accepted that $20 \%-50 \%$ of patients in intensive care units would contract ventilator-associated pneumonia and several would die. "We thought that was common and okay."

But after interventions were developed and protocols put in place, rates of this pneumonia dropped from a baseline of 19.88 to 3.76 per 1000 ventilator days after only 13 months, Hassen told an international forum earlier this year.

Gardam is adamant that jurisdictions shouldn't just establish guidelines and reporting requirements "and then walk away and say it's done. Multiple strategies must be used and one on its own won't do anything."

For example, hospitals can improve handwashing routines, but that will have little impact unless accompanied by other measures, such as enhanced institutional cleaning regimes, he says.

Gardam oversees Ontario's three mobile multidisciplinary troubleshooting teams that respond to invitations from hospitals with high rates or outbreaks of hospital-acquired infections.

But there are 157 hospitals in the province and "even if we visit 10 this year because the rates are high and they want to control rates, the vast majority of $C$. difficile will be at the other 147 hospitals, so it is a bit like a drop in the bucket."

The real potential for change lies in behavioural change among health care workers, he says. "That is a bigticket item."

Responsibility for infection control has traditionally rested with infection 
prevention and control staff in hospitals. But while those professionals are good at assessing and tracking outbreaks and recommending measures to be taken, "they are not change agents per se," and you cannot lower rates "by edict," adds Hassen.

In Ontario, monthly rates have hovered around $0.27-0.39$ per 1000 patient-days since public reporting of C. difficile commenced in August 2008. Rates, including those for individual hospitals, are posted online (www .health.gov.on.ca/patient_safety/media/ cdad/cdad_med.html).

"I definitely want rates to come down, but right now the fact that rates haven't gone up is good," says Gardam.

In Quebec, $C$. difficile rates dropped dramatically down to pre-epidemic levels since the province instituted a surveillance program five years ago.

"It took us five years to come back to our baseline level. I guess the key thing is that it is a slow process," says Dr. Charles Frenette, chair of Quebec's surveillance program.

The rate for the four-month period from December 2008 to March 2009 was 6.9 per 10000 patient-days, according to the latest figures published by the Institut National de Santé Publique du Quebec. By comparison, it was 11.7 from 2004-2007.

In its efforts to bring down rates, the province has benefited from "a massive increase in the workforce of infection control professionals," says Frenette.

\section{Union says contract cleaning problematic}

Before a deadly Clostridium difficile outbreak in 2008, contract cleaners at the Nanaimo General Hospital in British Columbia were using a cleaning solution 100 times weaker than recommended. At the peak of the outbreak, they were not even cleaning the emergency department, according to an official report.

The ineffective cleaning solution "was clearly a contributing factor to the propagation" of the outbreak and the emergency department was "a possible amplifying area" that should have been cleaned twice a day because of the volume of patients and routine overcrowding, stated the report on the outbreak by the BC Centre for Disease Control.

"There were insufficient numbers of cleaning staff to meet the basic daily needs of the facility and they were not adequately trained ... [or] able to meet the increased demand for environmental cleaning that is required to control an outbreak," concluded the report.

Cleaning is as crucial as handwashing to infection control, but it "has received far less attention and resources," the Canadian Union of Public Employees (CUPE) stated in its January 2009 report Healthcare Associated Infections: A Backgrounder.

Effective hospital cleaning is a key component of infection control, but budget cuts and outsourcing have seen the proportion of hospital budgets devoted to support staff drop from $26 \%$ in 1976 to $16 \%$ in 2002, according to the report.

The Nanaimo outbreak, associated with eight confirmed deaths, led the BC Centre for Disease Control to recommend that the hospital review its cleaning contract with the Compass Group and audit services to ensure appropriate staffing levels and cleaning protocols.

But the CUPE report argued for an end to the contracting out of cleaning services since it leads to staff cuts, lower wages, a higher turnover rate and "a rift between clinical and support services. High turnover, poor training, and the breakdown in teamwork are factors in HAI [hospital acquired infection] outbreaks."

The union report cited a 2007 auditor general of British Columbia report which stated that contracting out of cleaning services at BC Children's and Women's Health Centre "was initially acceptable, but that it began to slip when the cleaning staff continually changed."

Private for-profit cleaning companies face a conflict of interest between profits and hygiene standards, the CUPE report concluded.

Quebec has also had success in cutting the number of methicillin-resistant Staphylococcus aureus cases, to 350 in 2008 from 700 in 2003, he adds.

In British Columbia, although the province's auditor general recommended centralized reporting for all health care associated infections, $C$. difficile is not currently reportable, says Bruce Gamage, manager of BC's infection control network.

But after establishing standard definitions, the network began collecting $C$. difficile rates from hospitals and health authorities on a voluntary basis and will publish its first report later this month, he adds.

While BC studied the reporting mechanisms in Ontario and Quebec "to see what works and what doesn't," it does not favour public reporting of hospital rates, Gamage says. "Our concern is that data is there without interpretation - you could have a large tertiary care hospital, where the sickest people have the most complicated procedures, compared to a small community hospital."

$C$. difficile has been reportable in Manitoba since April 2005, and in $2007-$ 2008 , the province had a rate of 1.2 per 1000 admissions and 1.7 per 10000 patient-days.

Limited national data is available from the Canadian Nosocomial Infection Program, but what data exists is usually at least two years out of date. The latest annual report, for 2007 , has data from 47 hospitals across the country and provides rates only by region, not by province. According to the report, Ontario and Quebec had the highest rate per 1000 admissions (4.96), followed by the four western provinces (4.08) and the Atlantic provinces (3.44).

"Ideally, in the future, when we get health surveillance systems integrated, we could have a coordinated national data set," says Dr. Kumanan Wilson, Canada Research Chair in Public Health Policy at the University of Ottawa in Ontario.

C. difficile is highly infectious but doesn't cross borders rapidly, so the need for national reporting isn't pressing, Wilson adds. - Ann Silversides, CMAJ

DOI:10.1503/cmaj.109-3056 